\title{
Cyclone Hard X-Ray Observatory
}

Steven E. Boggs, Robert P. Lin, Brian R. Dennis, Norman W. Madden, Peter von Ballmoos, et al.

Steven E. Boggs, Robert P. Lin, Brian R. Dennis, Norman W. Madden, Peter von Ballmoos, Knud Thomsen, Gordon J. Hurford, Kevin C. Hurley, David M. Smith, Pierre Jean, Juergen Knoedlseder, Robyn M. Millan, "Cyclone Hard X-Ray Observatory," Proc. SPIE 4140, X-Ray and Gamma-Ray Instrumentation for Astronomy XI, (13 December 2000); doi: 10.1117/12.409159

Event: International Symposium on Optical Science and Technology, 2000, San Diego, CA, United States 


\title{
The Cyclone Hard X-Ray Observatory
}

\author{
Steven E. Boggs ${ }^{a, b}$, Robert P. $\operatorname{Lin}^{b}$, Brian R. Dennis ${ }^{c}$, Norman W. Madden ${ }^{d}$, \\ Peter von Ballmoos ${ }^{e}$, Knud Thomsen ${ }^{f}$,Gordon J. Hurford ${ }^{b}$, Kevin C. Hurley ${ }^{b}$, David M. Smith ${ }^{b}$, \\ Pierre Jean $^{e}$, Jurgen Knoedlseder ${ }^{e}$, Robyn M. Millan ${ }^{b}$ \\ ${ }^{a}$ Space Radiation Laboratory, California Institute of Technology, Pasadena, CA 91125, USA \\ ${ }^{b}$ Space Sciences Laboratory, University of California, Berkeley, CA 94720, USA \\ ${ }^{c}$ NASA/Goddard Space Flight Center, Code 682, Greenbelt, MD 20771, USA \\ ${ }^{d}$ Lawrence Berkeley National Laboratory, University of California, Berkeley, CA 94720, USA \\ ${ }^{e}$ Centre d'Etude Spatiale des Rayonnements, CNRS/UPS, BP 4346, 31029 Toulouse Cedex, France

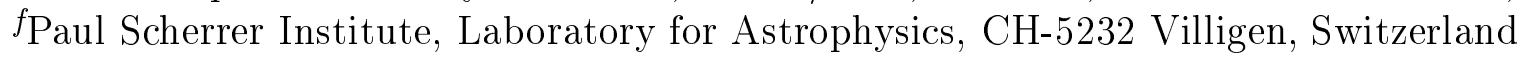

\begin{abstract}
In response to the recent NASA-SMEX Announcement of Opportunity, our collaboration proposed Cyclone, the Cyclotron/Nuclear Explorer. Cyclone is a broadband pointed astrophysical observatory, combining the highest spectral resolutions $(E / \Delta E \sim 30-300)$ and angular resolutions (15") achieved in the optimized hard X-ray range (10-200 keV). The instrument consists of 19 co-aligned rotation modulation collimator (RMC) telescopes, each with a high spectral resolution, 6-cm diameter germanium detector $(\mathrm{GeD})$ covering energies from $3 \mathrm{keV}$ to $600 \mathrm{keV}$. Both the optics and detectors are actively shielded with 15-mm BGO to gain low background and high sensitivity to astrophysical sources. A 550-km altitude, circular equatorial orbit also minimizes background. Building strongly upon instrumental heritage from the High-Energy Solar Spectroscopic Imager (HESSI) program, Cyclone would be ready for launch by September 2003. The instrument design and expected performance are discussed, as well as a brief overview of scientific goals.
\end{abstract}

Keywords: high energy astrophysics; x-ray/gamma-ray detectors; satellite missions

\section{INTRODUCTION}

High-resolution observations of astrophysical nuclear line emission is the highest priority scientific goal for future hard $\mathrm{X}$-ray/soft $\gamma$-ray missions. ${ }^{1}$ Almost all of the important isotopic decay chains produced in supernova nucleosynthesis ${ }^{2}$ result in nuclear line emission in the hard X-ray range (10-200 keV) as well as the soft $\gamma$-ray range $(0.3-10 \mathrm{MeV})$, e.g., ${ }^{44} \mathrm{Ti},{ }^{56} \mathrm{Ni},{ }^{57} \mathrm{Ni},{ }^{26} \mathrm{Al}-{ }^{60} \mathrm{Fe}$. Cyclone is optimized to perform narrow FOV $\left(\sim 1^{\circ}\right)$, targeted observations in the 10-200 keV energy band, achieving unprecedented hard X-ray spectral resolutions $(E / \Delta E \sim 30-300)$, angular resolutions (15"), and nuclear line sensitivities (Table 1). Cyclone is an order of magnitude more sensitive than Integral/SPI to nuclear line emission at hard X-ray energies, and over 4 times more sensitive in hard X-rays than Integral/SPI at its peak soft $\gamma$-ray sensitivity. Cyclone achieves hard X-ray spectral resolution factors of $\sim 3$ better than Integral/SPI. The improvements in hard X-ray performance over Integral/SPI result from (i) the use of GeDs optimized for energies below $200 \mathrm{keV}$, and (ii) Cyclone's decreased background due to a much narrower FOV and equatorial orbit. For imaging, Cyclone gains 50 times better angular resolution than the imager Integral/IBIS, and 500 times better than Integral/SPI. Cyclone will map the sites of $68 / 78 \mathrm{keV}^{44} \mathrm{Ti}$ nuclear line emission (Figure 1) in young supernova remnants (SNRs), which have typical diameters <10' (Cas A 4', Kepler 3.4', Tycho 8').

Cyclone is also a powerful observatory for the study of neutron stars and black holes. Cyclone's spectral resolution will resolve, for the first time, the electron cyclotron lines in magnetized neutron stars, and potentially discover proton cyclotron lines in magnetars. Timing of every photon to $1 \mu \mathrm{s}$ will help explore the detailed accretion structure and magnetic field geometry in these objects. Sub-arcminute imaging of nonthermal emission in Galactic supernova remnants and extragalactic jets will pinpoint and characterize enigmatic sites of particle acceleration. Spatially resolved spectroscopy of crowded Galactic fields will detect and classify new sources, including the massive black hole at the center of our Galaxy. A systematic survey of infrared-identified candidates will reveal obscured active galactic nuclei, quantifying their contribution to the $30 \mathrm{keV}$ peak in the cosmic X-ray background.

Further author information: Send correspondence to S.E.B., E-mail: boggs@srl.caltech.edu 

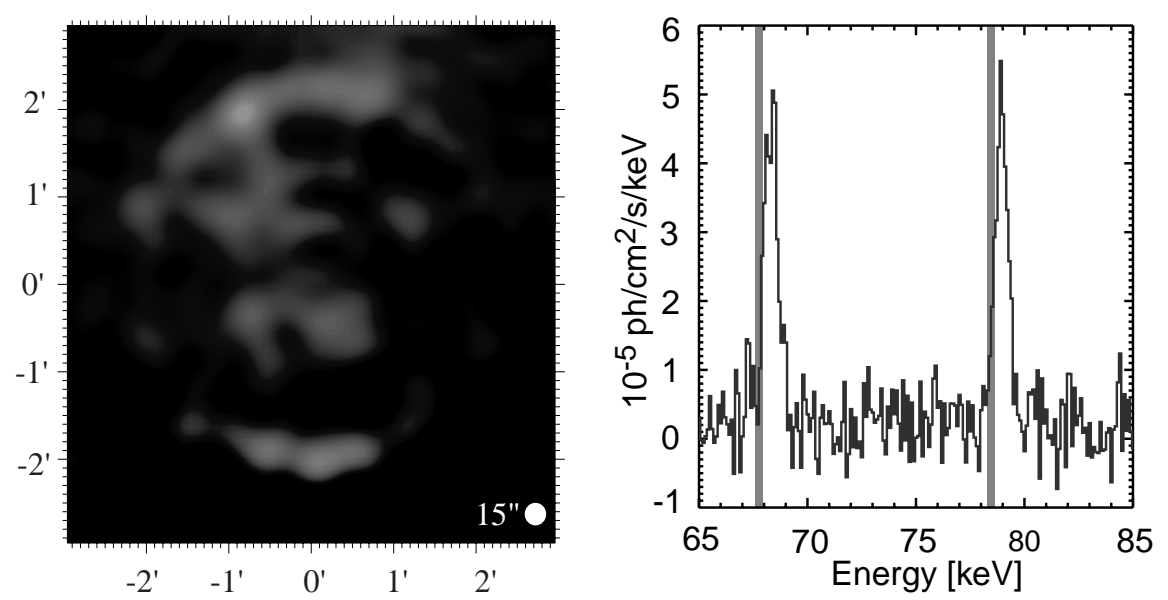

Figure 1. Simulated $10^{6} \mathrm{~s}$ Cyclone observation of the Cas A SNR ${ }^{44} \mathrm{Ti}$ emission. At left is the CLEANed image of the 68/78 keV emission, assuming a clumpy, axisymmetric distribution. Also shown is the 15" angular resolution. At right is the integrated line spectrum, measured at $\sim 50 \sigma$. The energy resolution $(2100 \mathrm{~km} / \mathrm{s} \mathrm{FWHM})$ is indicated by the grey stripes at the unshifted line energies. Cyclone will also provide continuum spectra (3-200 keV) for individual features.

Launch in 2003 would maximize Cyclone's scientific impact. Integral (2002) will have been observing for over a year, providing many new sources for detailed study. Swift (2003) and MAXI (2003) will provide powerful all-sky monitoring capabilities. Chandra and XMM will be fully active, and Cyclone will provide a unique hard X-ray complement with its broad energy band (extending down to $\sim 3 \mathrm{keV}$ ) and high angular resolution. During its 2-3 year mission Cyclone will perform 100-200 detailed source observations. For a 3-year mission, Cyclone will perform coordinated observations with GLAST (2005). Rapid, direct access by the astrophysics community to the Cyclone data and software, combined with a large Guest Observer Program ensures the optimal scientific return from the mission.

Table 1. Cyclone Mission Characteristics

\begin{tabular}{|l|l|}
\hline Energy Range & $10-200 \mathrm{keV}$ Optimized, 3-600 keV Full \\
\hline Energy Resolution & $350 \mathrm{eV}<20 \mathrm{keV}, 550 \mathrm{eV} @ 78 \mathrm{keV}(\mathrm{FWHM})$ \\
\hline Angular Resolution/Range & $15^{\prime}-13.5^{\prime}(\mathrm{FWHM})$ \\
\hline FOV & $\sim 1^{\circ}$ \\
\hline Total Detector Area & $540 \mathrm{~cm}^{2} \mathrm{GeDs}$ \\
\hline Effective Photopeak Area & $105 \mathrm{~cm}^{2} @ 78 \mathrm{keV}$ \\
\hline Narrow Line Sensitivity & $4 \times 10^{-6} \mathrm{ph} / \mathrm{cm}^{2} / \mathrm{s} @ 78 \mathrm{keV},\left(5 \sigma, 10^{6} s\right)$ \\
\hline Point Source Sensitivity & $0.12 \mathrm{mCrab}(10-100 \mathrm{keV}),\left(5 \sigma, 10^{6} s\right)$ \\
\hline \hline Orbit & Equatorial LEO $(550 \mathrm{~km})$ \\
\hline Spacecraft & Spin Stabilized, $60-\mathrm{s} \mathrm{Rotation} \mathrm{Period}$ \\
\hline Pointing Requirements & $\leq 0.5^{\circ}$ \\
\hline Aspect Performance & $2^{\prime \prime}$ Pitch \& Yaw, 20" Rotation \\
\hline Launch Date & September 2003 \\
\hline Mission Lifetime & 2 years Nominal, $\geq 3$ years Desired \\
\hline
\end{tabular}




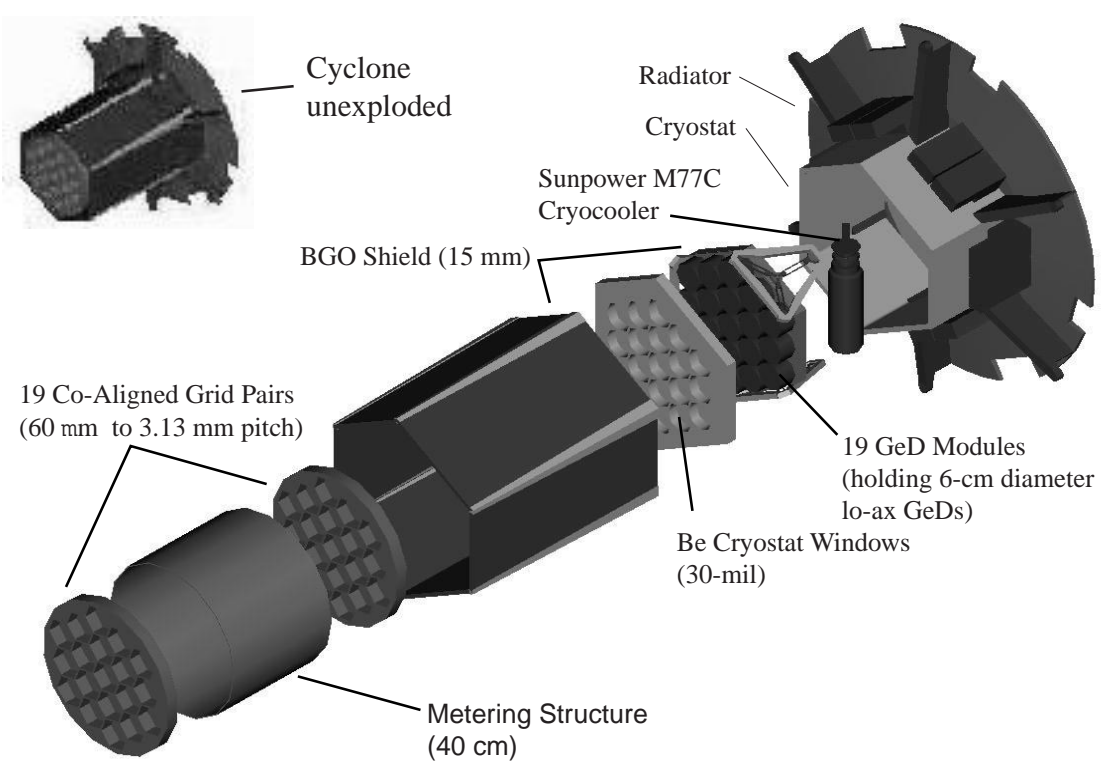

Figure 2. Exploded view of the Cyclone scientific instrument showing the major components. The unexploded view is shown in the upper left corner (courtesy P. Turin, SSL/UCB).

\section{TELESCOPE DESIGN}

Cyclone utilizes an array of 19 co-aligned Rotation Modulation Collimator (RMC) telescopes (Figure 2), each consisting of a pair of widely separated 1-D opaque grids mounted in front of a hard X-ray GeD. The GeDs are mounted in a single cryostat, and cooled to $77 \mathrm{~K}$ by a space-qualified long-life Sunpower M77C Stirling-cycle cryocooler to achieve and maintain the highest spectral resolution of any presently available hard X-ray detector. As the spacecraft rotates, the RMCs convert the spatial information from the FOV into temporal modulation of the photon counting rates of the GeDs. A bismuth germanate (BGO) scintillator shield encloses the bottom and sides of the GeDs and RMC grids to minimize background. The instrument electronics amplify, shape, and digitize the GeD and BGO signals, provide low-voltage power and GeD and BGO high voltage, format the data, and interface to the spacecraft electronics. The energy and arrival time of every photon together with aspect data are recorded in the instrument 1-Gbit memory and automatically telemetered within 24 hours. With these data, the hard X-ray images and spectra can be reconstructed on the ground (ex., Figure 1). Cyclone builds upon strong instrumental heritage from HESSI ${ }^{3,4}$ and commercially available components, allowing the observatory to be launched by September 2003.

\subsection{IMAGING SYSTEM}

Each grid in an RMC (Figure 3) is a planar array of equally spaced hard X-ray-opaque slats separated by transparent slits. The slits of each pair of grids are parallel to each other and their pitches (p) are identical. The photon transmission through the grid pair depends on the direction of the incident photons. For slits and slats of equal width, the transmission is periodically modulated from zero to $50 \%$ and back to zero as the angle (orthogonal to the slits) between the source and collimator axis is changed by $\mathrm{p} / \mathrm{L}$ where $\mathrm{L}$ is the separation between grids. For Cyclone, using fixed grids on a rotating spacecraft varies this angle. The detector records the arrival time and energy of individual photons, allowing the modulated count rate to be determined as a function of rotation angle (Figure 4). The detector itself does not need any spatial resolution and so can be optimized for high sensitivity and fine energy resolution. For complex sources, and over small rotation angles, the amplitude and phase of the waveform provides a direct measurement of a single Fourier component of the angular distribution of the source (e.g., Ref. 5). Different Fourier components are measured at different rotation angles and with grids of different pitches. In practice, observations from many rotations can be combined, whether or not there is good statistics in any single rotation or modulation cycle. 


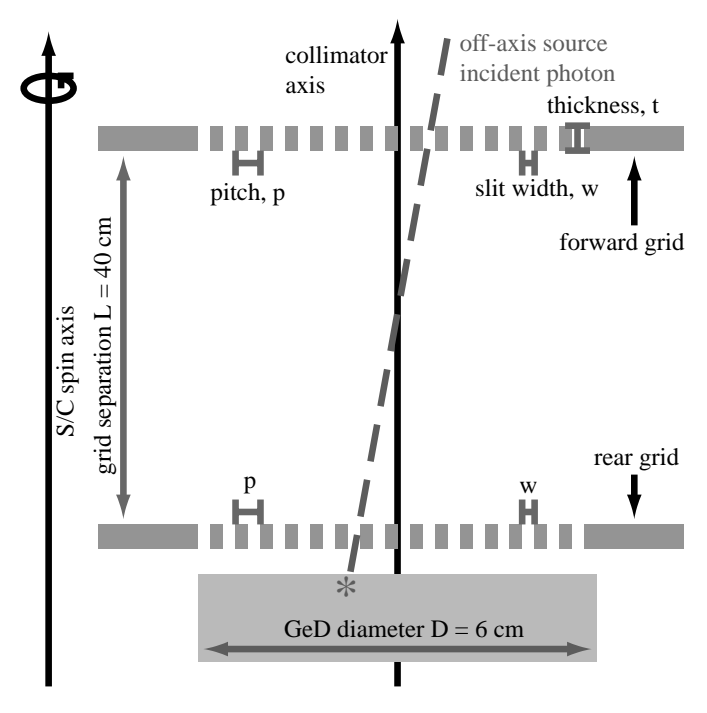

Figure 3. Sketch of the instrument parameters that define Cyclone's imaging capabilities (Section 2.1).

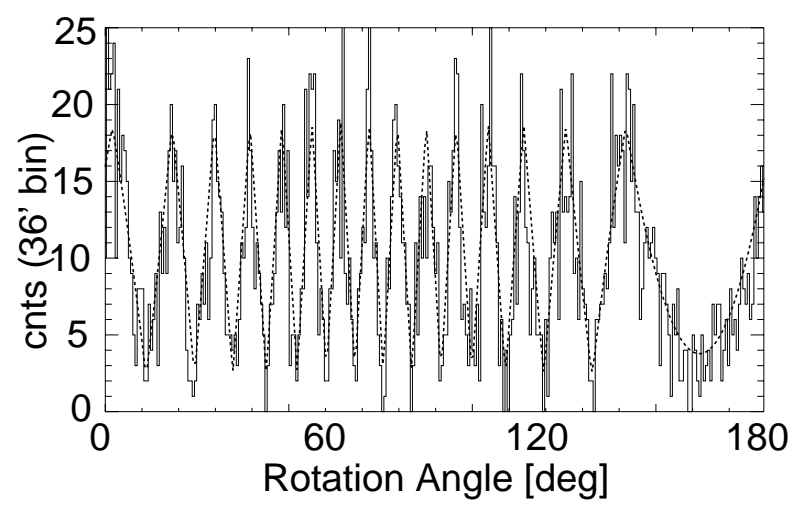

Figure 4. Example 10 minute integration of a 1 Crab (10-100 keV) source plus background with the 2.89' resolution grids, showing the grid modulation (dotted line) and measured counts (solid histogram). 


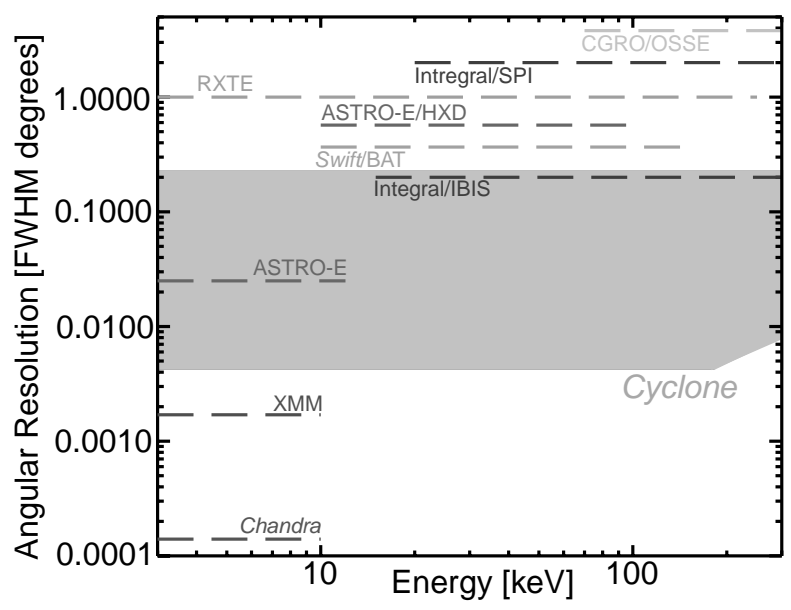

Figure 5. Cyclone's range of angular resolutions (15"-13.5') compared with current and future missions (Astro-E included for comparison). Cyclone's finest resolution is 50 times better than Integral/IBIS.

For Cyclone, the separation between grids is $L=40 \mathrm{~cm}$ and the grid pitches range from $60 \mu \mathrm{m}$ to $3.13 \mathrm{~mm}$ in steps of $3^{1 / 5}$, resulting in angular resolutions (defined as $\mathrm{p} /(2 \mathrm{~L})$ ) that are spaced logarithmically from 15 " to 13.5' (Figure 5). Thus sources will be imaged over a wide range of angular scales. Diffuse sources larger than 13.5' in extent are not imaged. Multiple smaller sources within the $\sim 1^{\circ}$ FOV are imaged regardless of their separation. By arranging the grids so that neighboring pairs do not have parallel slit directions, each detector is effectively collimated from photons incident through the aperture of its neighbors. For a typical imaging situation, the 19 RMCs measure amplitudes and phases of over $10^{3}$ Fourier components in a half rotation $(30 \mathrm{sec})$, thus ensuring that the image quality is limited by statistics, and not by the finite number of measured Fourier components. Detailed high-resolution X-ray spectra can be obtained for each feature in the image, thus providing true high-resolution imaging spectroscopy.

Molybdenum (Mo) was selected as the grid material for its stopping power, activation properties, and ease of fabrication. The grids range in thickness from $8.0 \mathrm{~mm}$ for the coarsest grids to $1.9 \mathrm{~mm}$ for the finest. The FOV of a grid is determined by the ratio of its pitch to thickness. The grid thicknesses were chosen to maintain over $95 \%$ modulation efficiency up to $80 \mathrm{keV}$ for all the grids, and over $85 \%$ modulation up to $200 \mathrm{keV}$ for the coarsest 10 grids (Figure 6). The technique developed by Thermo Electron Tecomet for HESSI will be used to fabricate all 38 grids. Sheets of Mo, as thin as or thinner than the slit width, are first etched with the required slit pattern and alignment holes. These sheets are stacked and bonded to produce a rugged grid that satisfies rigorous pitch and uniformity requirements at the few microns level. The grid properties and alignment will be calibrated to 1\% using the NASA/GSFC optical and X-ray characterization facilities.

\subsection{DETECTOR PLANE}

The Cyclone detector plane array consists of $19 \mathrm{GeDs}$ in individual modules mounted in a single cryostat, surrounded by an active anticoincidence shield, and cooled by a mechanical cryocooler (Figure 2). GeDs cover the entire hard Xray/soft $\gamma$-ray energy range with the highest spectral resolution of any readily available detector technology (Figures 7 \& 8). Cyclone utilizes commercially produced PerkinElmer ORTEC (formerly EG\&G ORTEC) LO-AX GeDs, 6-cm diameter and 2.0-cm thick, with quasi-hemispherical electrode configurations for very low capacitance. The $\mathrm{GeD}$ thickness of $2.0 \mathrm{~cm}$ provides maximum efficiency up to $200 \mathrm{keV}$, while avoiding excess volume to minimize activation background. The total active detector area is $540 \mathrm{~cm}^{2}$. The GeD outer surface is implanted with a thin $(0.3 \mu \mathrm{m})$ boron layer to provide both a $\mathrm{P}+$ electrode and a front surface transparent down to $\sim 3 \mathrm{keV} \mathrm{X}$-rays. The inner bore is diffused with lithium $(500 \mu \mathrm{m})$ to form the $\mathrm{N}+$ contact.

The GeDs are mounted in a shorter version of the detector modules used for HESSI, which have been successfully vibration tested and are fully capable of withstanding a Pegasus XL launch. The input FET is mounted close to the $\mathrm{GeD}$ for noise immunity, but is thermally tied to the outer driven shield to provide operating temperatures of $\sim 160 \mathrm{~K}$. The GeD modules are mounted on an aluminum coldplate, and enclosed by two thin IR radiation and 


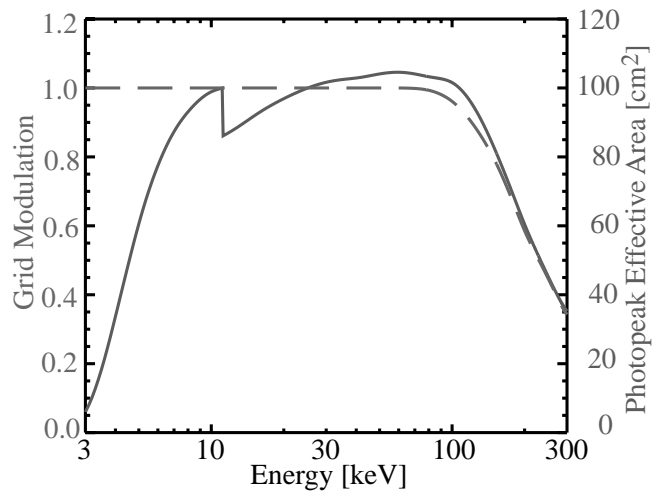

Figure 6. Cyclone's photopeak effective area (solid line), including the effects of detector efficiency, window transparency, and grid transmission. Also shown is the average grid modulation (dashed line).

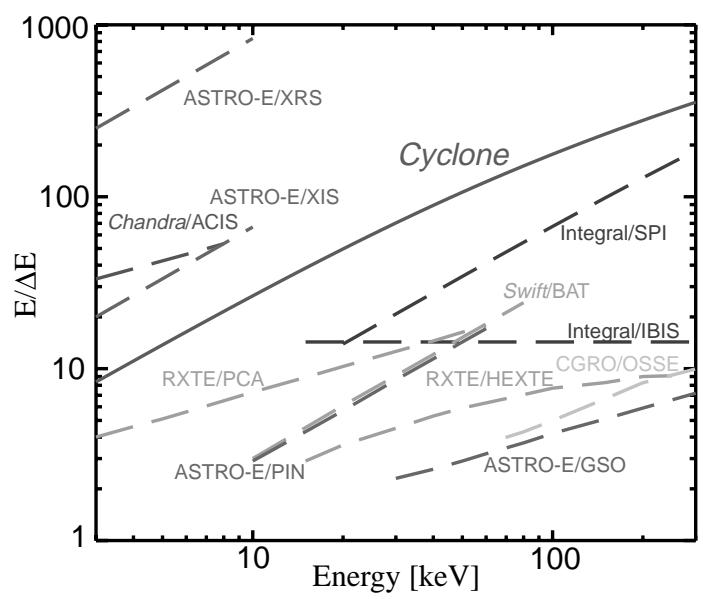

Figure 7. Cyclone's spectral resolution (FWHM), with factors of $\sim 3$ improvement over Integral/SPI by optimizing the GeDs for hard X-ray energies. 


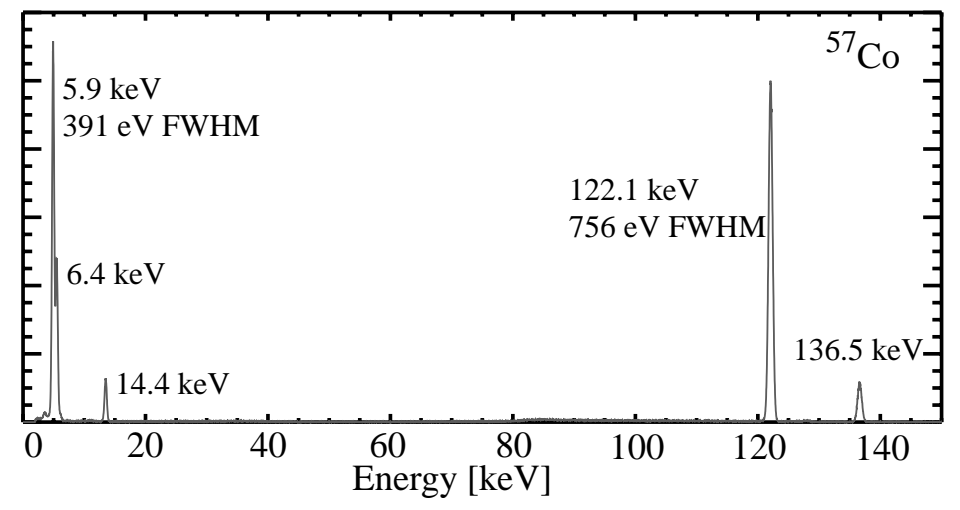

Figure 8. Laboratory spectrum of a LO-AX GeD. Cyclone flight electronics will achieve even lower electronic noise than the laboratory electronics used here (spectrum courtesy T. W. Raudorf, PerkinElmer Instruments Inc.).

anti-contamination shields. The shielded $\mathrm{GeD} /$ cold plate assembly is surrounded by four 7-layer thermal blankets, and supported inside the cryostat vacuum shell by low conductivity thermal mounts. A 0.25 -mm beryllium window in the cryostat in front of each GeD allows ample transparency for observations down to $3 \mathrm{keV}$.

Each GeD is biased at $4-5 \mathrm{kV}$ by a separate adjustable high-voltage power supply mounted just outside the cryostat. Photons interacting in a GeD generate charge pulses, which are collected and amplified by a transistorreset preamplifier (developed by LBNL for HESSI) to provide the best resolution and to eliminate problems associated with pole-zero cancellation. An advanced 4-terminal type FET, developed by Greshem Scientific, has 3-4 times the gain and half the capacitance of available commercial units. The preamplifier signal goes to dual signal processing chains. The high resolution "slow" chain, optimized for spectral resolution, consists of a 4-pole shaping amplifier with a peaking time of $15 \mu \mathrm{s}$ for $\sim 350 \mathrm{eV}$ FWHM electronic resolution. This is followed by a peak stretcher, and a 16-bit successive approximation analog-to-digital converter. The upper 13 bits provide 8192 channels from $3 \mathrm{keV}$ to $600 \mathrm{keV}$ with $70 \mathrm{eV} /$ channel. The high rate "fast" chain consists of a double delay-line shaper generating a triangular pulse of $0.2-\mu \mathrm{s}$ duration. The fast signal is used to detect GeD-GeD and GeD-shield coincidences, and for pulse pileup rejection. The threshold for this fast chain is low enough that germanium background line photons at 53.4 and $13.3 \mathrm{keV}$, which follow each other with a $2.9-\mu$ s half-life, can be $>90 \%$ rejected. Unresolved events are electronically combined into a narrow line at $66.7 \mathrm{keV}$.

The GeDs are cooled on-orbit by a single Sunpower M77C Stirling-cycle cooler (Figure 2). These coolers have a linear compressor with a gas-bearing supported piston and displacer, in line with a counterbalancer. Through an extensive series of tests, GSFC's Cryogenics and Fluids Branch has demonstrated that the gas bearings prevent touch contact during operation of the cooler, eliminating all wear. All components are designed for a minimum of 50,000 hours of operation. Detailed thermal models (developed for and tested with the HESSI cryostat), taking into account the FETs, wiring, thermal blankets, support structure, etc., show that a single Sunpower M77C cooler will cool the GeDs to $77 \mathrm{~K}$ with $60 \mathrm{~W}$ of power.

The active shield, crucial for reaching Cyclone's low background rates, is composed of seven slabs of BGO, $\sim 15 \mathrm{~mm}$ average thickness, arranged in a hexagonal well surrounding both the detector cryostat and the imaging optics (Figure 2). The side slabs are tapered, and the large hermetically sealed bottom shield is located inside the cryostat to reduce mass. Each BGO segment will be viewed by one or more pairs of photomultiplier tubes (PMTs), coupled to a low-power, long-life electronics chain with a threshold of $\sim 60 \mathrm{keV}$ (developed by LBNL for the GammaSphere Cyclotron instrument). Each PMT of the pair will operate from a different bias supply, so a failure in one of the two bias supplies will not create a hole in the shield but only raise the threshold by a factor of 2 . GeD pulses occurring within $1 \mu s$ of a shield pulse are rejected. Total dead time is estimated (from BATSE measurements) to be a few percent. 


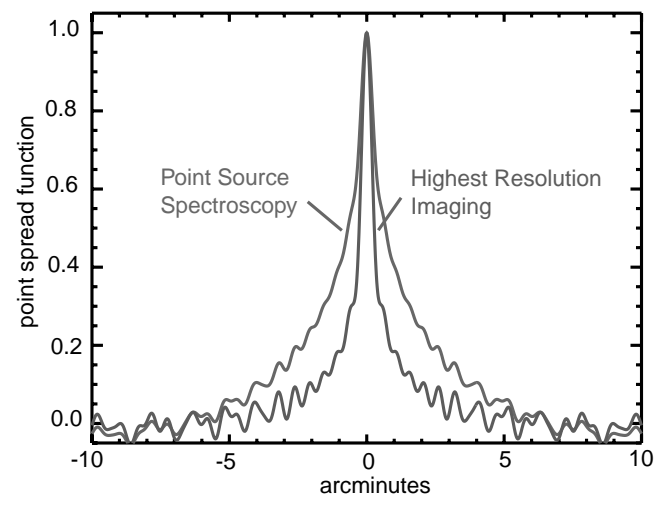

Figure 9. The Cyclone point spread function for two different scientific weightings.

\section{INSTRUMENT PERFORMANCE}

\subsection{Detector Efficiency and Grid Modulation}

Figure 6 shows the overall photopeak effective area for Cyclone, determined by convolving the energy-dependent GeD photopeak efficiency and the angular- and time-averaged transmission of the grid pairs. Also shown is the average modulation efficiency, defined as the modulation amplitude of the grids for a typical off-axis source location, compared to perfect, opaque grids of zero thickness and equal slit and slat widths. The effective area is optimized $\left(>100 \mathrm{~cm}^{2}\right)$ in the range 6-120 keV. Adequate area remains for performing X-ray imaging and spectroscopy down to $3 \mathrm{keV}$. The GeDs and grids were designed to have high efficiency up to the $158 \mathrm{keV}{ }^{56} \mathrm{Ni}$ decay line, and the GeD response remains significant above $200 \mathrm{keV}$.

\subsection{Energy Resolution}

The low-capacitance electrode configuration of the LO-AX GeDs, together with the advanced FET and analog electronics optimized for resolution, provide an electronic resolution of $\sim 350 \mathrm{eV}$ FWHM. The energy-dependent photon resolution is given by: $\Delta E^{2}=(.350)^{2}+2.1 \times 10^{-3} E$ FWHM, where $\Delta E, E$ are in units of keV. Figure 7 shows $E / \Delta E$ for Cyclone, which has the highest spectral resolution of any instrument covering 10-200 keV, exceeding Integral/SPI by factors of $\sim 3$ at the $68 / 78 \mathrm{keV}$ lines of ${ }^{44} \mathrm{Ti}$. Figure 8 shows a measured laboratory spectrum taken with a LO-AX GeD.

\subsection{Angular Resolution}

The 19 RMCs provide sensitivity to Fourier components from 15" to 13.5' (Figure 5), in steps of $3^{1 / 5}$. The highest resolution was chosen to centroid point-sources to $<5^{\prime \prime}$ for identifying obscured AGN, to resolve knot-like structures in typical SNRs (e.g., Cas A is 4' diameter, Figure 1), and to separate source-confused regions, such as SGR A* at the Galactic Center. Localization of most point sources will be limited by the 2" uncertainty of the aspect system. The coarsest resolution of 13.5' was chosen to image diffuse emission in young SNRs. The point-spread function (PSF) depends on how the 19 elements are weighted in the data analysis, which in turn depends on the scientific questions being asked. Figure 9 shows two PSFs, one optimized for point-source separation, the other for point-source spectroscopy. This ability to adjust analysis techniques to optimize the scientific output is an important aspect of Cyclone's strength and flexibility in addressing many diverse scientific questions.

\subsection{Background}

Every effort has been made to understand and minimize the Cyclone background (Figure 10). (1) Cyclone will be in a high geomagnetic cutoff, equatorial low Earth orbit, greatly reducing the hadronic induced background components. (2) Both the GeDs and the grids are actively shielded, to reduce both incident $\gamma$-ray backgrounds (cosmic, atmospheric, satellite), and unvetoed induced prompt $\gamma$-ray emission from the grids. (3) Backgrounds for GeD spectrometers in deep-well BGO active shields are extremely well studied (Refs. 6-10), especially with the intense modeling effort being performed for Integral/SPI. (4) The GeD thickness is kept $\leq 2 \mathrm{~cm}$ to minimize 


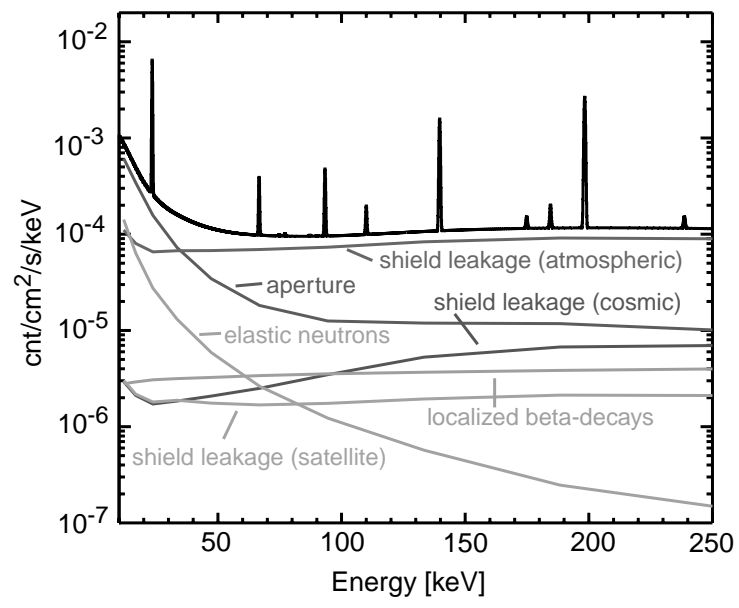

Figure 10. Cyclone background calculation, assuming a $550 \mathrm{~km}$ circular equitorial orbit (Section 3.4).

internal activation. (5) The choice of molybdenum grids eliminates activation lines in crucial nuclear line regions (unlike tungsten). (6) With high spectral resolution GeDs, energy regions including induced background lines can be excluded from spectral analysis to improve sensitivity. (7) RMC imaging modulates between source and background on timescales much faster than the spacecraft spin period (60 s) and orbital background variations (tens of minutes), so that background variations are effectively removed. (8) Fast pulse pileup rejection suppresses the induced 53.4/66.7 $\mathrm{keV}$ line complex in the GeDs.

The cosmic, atmospheric, and satellite background components were modeled using a GEANT Monte Carlo simulation of the instrument, including active and passive materials. The internal background components were extrapolated to Cyclone's orbit and shielding from simulations for a number of satellite and balloon instruments including Integral/SPI. Those calculations generally agree with observations to within $<20 \%$. Below $30 \mathrm{keV}$, the background is dominated by the well-known cosmic aperture flux. At higher energies, shield leakage of induced atmospheric $\gamma$-rays dominates. Cyclone background calculations are presented in Figure 10; these were used with the efficiency calculations to determine the sensitivity estimates presented in Figures $11 \& 12$, as well as the science simulations (ex., Figure 1).

\subsection{Dynamic Range}

Besides the statistical and point response calculations we must consider potential performance limitations imposed by systematic uncertainties, such as imperfect knowledge of the RMC angular response, and the effects of time-varying background. A relevant measure is the dynamic range of the image, defined as the ratio of the brightest to the faintest believable feature in an image. A conservative estimate would require knowledge of the grid modulation at the 1\% level in order to achieve imaging with 100:1 dynamic range. Experience with HESSI showed that preflight calibrations alone can define the modulation produced by each grid pair with an accuracy of $\sim 1 \%$. Techniques to exploit redundancies in the in-flight data to improve this by another factor of $\sim 3$ to 5 have been developed and will be confirmed with HESSI on-orbit observations. While we believe that 500:1 is achievable, a dynamic range of 100:1 is more than adequate to achieve all of the primary science goals.

\subsection{Sensitivity \& Systematic Uncertainties}

Cyclone sensitivities to narrow lines and continuum point sources are presented in Figures $11 \& 12$. Observations of narrow lines are relatively immune to systematic uncertainties in the background since they are seen with respect to the continuum immediately above and below them and so will always be limited purely by statistics. For backgrounddominated observations of continuum sources, systematic uncertainties for most hard X-ray $/ \gamma$-ray missions are on the order of $1 \%$. An RMC system has no moving parts, and the same detector reads source and background repeatedly and rapidly in succession, so it avoids systematics associated with off-target pointing, moving masses, or nonuniform response in a position sensitive detector plane. The modulation period for even the coarsest grids is much faster 


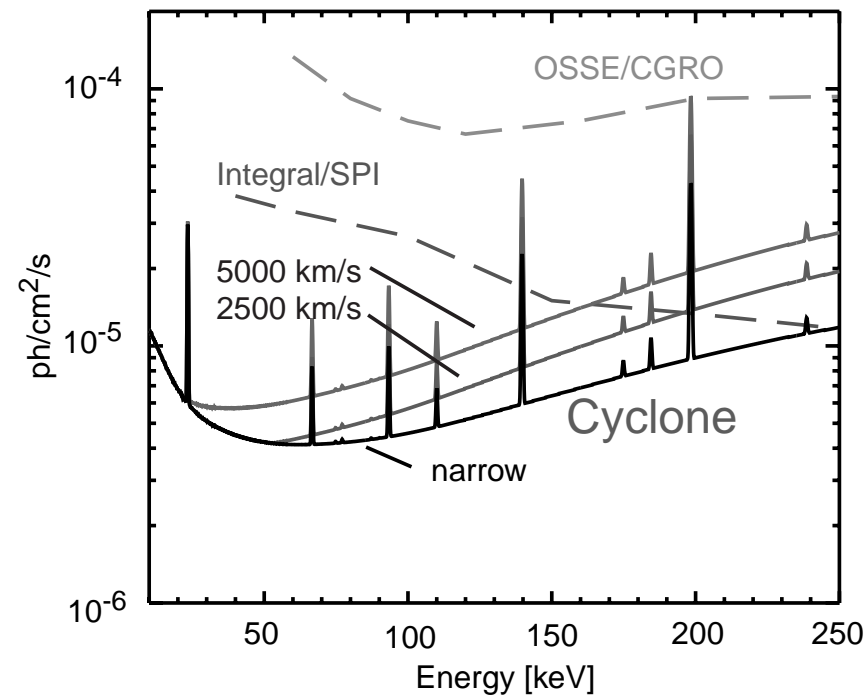

Figure 11. Cyclone's narrow line sensitivity $\left(5 \sigma, 10^{6} \mathrm{sec}\right)$, as well as the broad line sensitivities for $2500 \mathrm{~km} / \mathrm{s}$ and $5000 \mathrm{~km} / \mathrm{s}$ Doppler broadening. Shown for comparison are the narrow line sensitivities for Integral/SPI, and CGRO/OSSE.

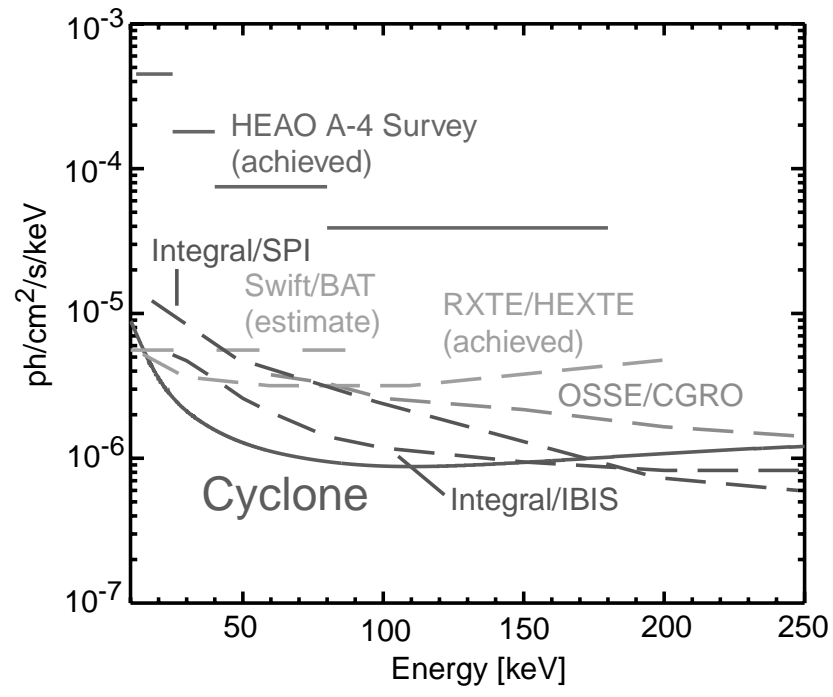

Figure 12. Cyclone's continuum sensitivity $\left(\Delta E=E / 2,5 \sigma, 10^{6} \mathrm{sec}\right)$. With its low background, Cyclone achieves improved sensitivities over much larger instruments.

than the spacecraft spin period, so that any effects of variations in the background with spin and orbital phase will be suppressed.

The task of extracting the modulated signal, which has a characteristic shape, from the time-dependent background is very similar to extracting the intensity of a pulsar of known frequency from a high-background instrument. The observations of pulsars with BATSE ${ }^{11}$ revealed pulsed signals down to about $0.2 \%$ of the background level. The modulation produced by the Cyclone's grids essentially makes every source a "pulsar" of known frequency. We are confident of reaching at least the $0.2 \%$ level of control of background systematics with Cyclone, which, as a shielded instrument in an equatorial orbit, will have a much less variable background than BATSE. The sensitivities given in 


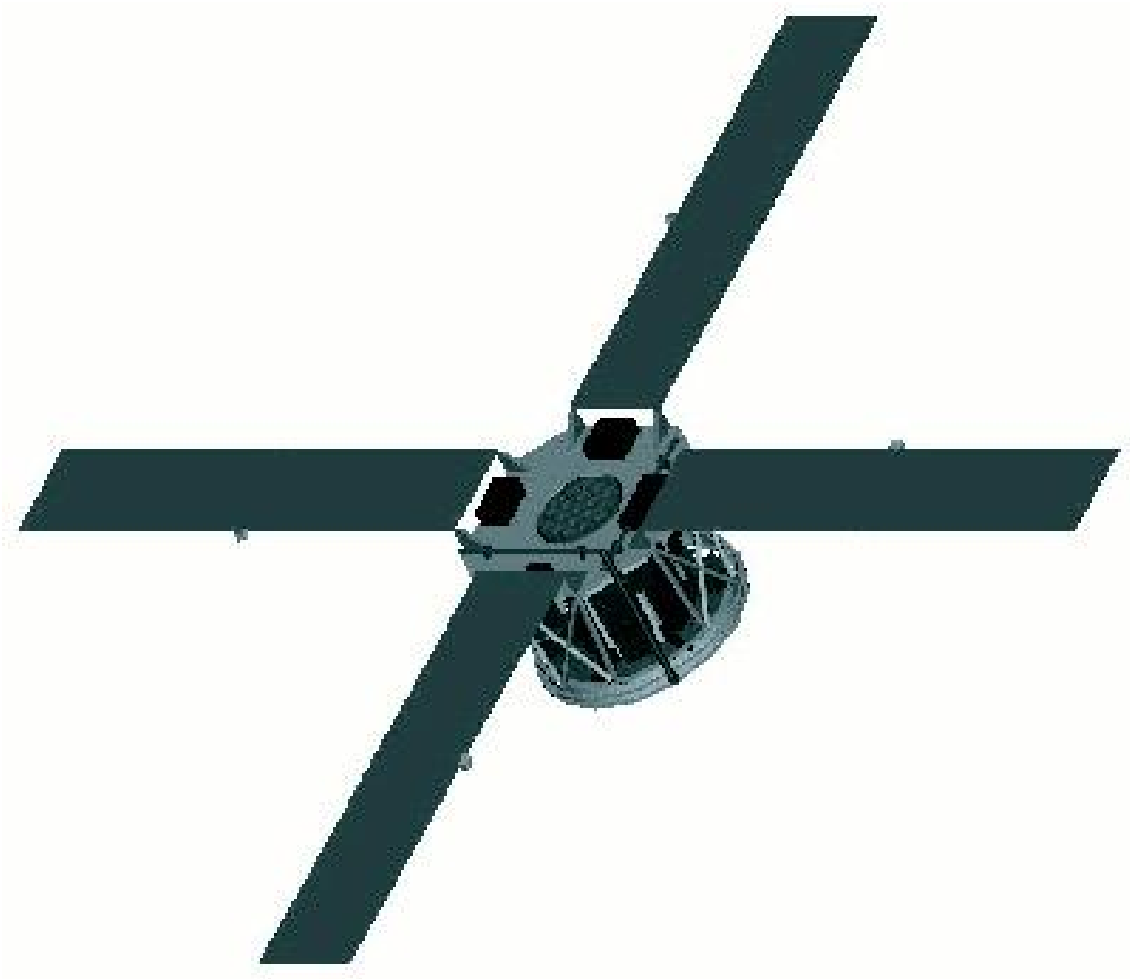

Figure 13. Cyclone's spin-stabilized spacecraft with the solar array deployed (courtesy P. Turin, SSL/UCB).

Figures $11 \& 12$, however, are achievable with $1 \%$ background systematic uncertainties.

\subsection{Timing}

Each photon interaction in Cyclone will be time-tagged with $1-\mu$ s resolution. For the study of transient and periodic X-ray sources, the effects of modulation on the time profiles must be removed. Techniques which exploit the redundancy of the multiple RMCs have been developed for observations of the Crab pulsar and transient solar sources with HESSI, and can be directly applied to Cyclone for dealing with such issues. Such techniques permit full decoupling of spatial and intrinsic temporal effects in the observed modulation, and full recovery of the location and photometrically correct waveform of transient and periodic sources. This will enable Cyclone to exploit its full potential for the study of transient and periodic sources.

\section{MISSION}

The Cyclone mission (Table 1) requires a simple spin-stabilized (60-s rotation) spacecraft (Figure 13), with the spin axis pointed at targets between $15^{\circ}$ and $62^{\circ}$ from the Sun (limited by solar array power), providing access to $88 \%$ of the sky over the course of the year. The $550-\mathrm{km}$ circular, equatorial orbit can be provided by a standard Pegasus XL launch. The instrument field of view is $\sim 1^{\circ}$, so spacecraft pointing is relaxed $\left(<0.5^{\circ}\right)$ and can be automated. Aspect is provided by redundant Advanced Stellar Compass (ASC) star trackers, developed at the Technical University of Denmark. The ASC can track attitude with 2" translational and 20" rotational precision at $4 \mathrm{~Hz}$ cadence, meeting Cyclone requirements. The instrument is on continuously. Typically, source pointings last 1-2 weeks, with the source eclipsed by the Earth $22-37 \%$ of each orbit, for pointing $62^{\circ}$ and $0^{\circ}$ off of the orbital plane respectively. All of the photon data collected for $24 \mathrm{hrs}$ (even for the strongest sources) can be stored in the instrument mass memory. Cyclone will pass over the Kourou ground station every orbit. Downlinks during $45 \%$ of the passes are sufficient to provide the required data downlink rate (100 Mbytes/day), thus minimizing tracking, data transmission, and operation. Consequently, Cyclone is planned for an automated store-and-dump operation, and normally no real-time 
access is required. Both the instrument and spacecraft are designed to operate autonomously for weeks at a time. With no expendables, Cyclone's lifetime could extend well beyond the 2-3 year nominal mission.

\section{SUMMARY}

Cyclone represents a major step forward in the evolution of hard X-ray/soft $\gamma$-ray astrophysics toward a narrow FOV, targeted observatory. The unprecedented combination of spectral resolution, angular resolution, and sensitivities make Cyclone a powerful observatory for studying nuclear line emission, SNRs, compact objects, the Galactic center, AGN, and other high energy sources. By addressing high-priority science while building upon readily available technologies and well-defined mission parameters (thanks to HESSI), Cyclone is ideally suited for the goals, timescales, and constraints of the NASA-SMEX program.

\section{ACKNOWLEDGMENTS}

S. Boggs would like to thank Caltech for support through the Millikan Fellowship Program. We would also like to thank R. Lingenfelter, D. Lamb, C. Thompson, and R. Duncan for participation in scientific discussions.

\section{REFERENCES}

1. Report of the NASA Gamma-Ray Astronomy Program Working Group (GRAPWG), Recommended Priorities for NASA's Gamma-Ray Astronomy Program 1999-2013, NP 1999 4 272 GSFC, p. 56, June 1999.

2. R. Diehl and F. X. Timmes, "Gamma-ray line emission from radioactive isotopes in stars and galaxies," PASP 110, pp. 637-659, 1998.

3. R. P. Lin, et al., "High-energy solar spectroscopic imager (HESSI) small explorer mission for the next (2000) solar maximum," Proc. SPIE 3442, pp. 2-12, 1998.

4. B. Dennis, et al., "High-energy solar spectroscopic imager (HESSI)," Proc. SPIE 2804, pp. 228-240, 1996.

5. T. A. Prince, et al., "Gamma-ray and hard X-ray imaging of solar flares," Solar Phys. 118, pp. 269-290, 1988.

6. N. Gehrels, "Instrumental background in balloon-borne gamma-ray spectrometers and techniques for its reduction," NIM A329, pp. 324-349, 1985.

7. A. J. Dean, F. Lei, and P. J. Knight, "Background in space-borne low-energy gamma-ray telescopes," Space Sci. Rev. 57, pp. 109-186, 1991.

8. N. Gehrels, "Instrumental background in gamma-ray spectromters flown in low Earth orbit," NIM A313, pp. 513-528, 1992.

9. J. E. Naya, et al., "Gamma-ray background lines in balloon and satellite-borne Ge spectrometers," Proc. SPIE 2806, pp. 472-482, 1996.

10. P. Jean, et al., "Performance of an Integral spectrometer model," Proc. 2nd Integral Workshop ESA SP-382, pp. 635-638, 1997.

11. L. Bildsten, et al., "Observations of accreting pulsars," ApJS 113, pp. 367-408, 1997. 